\title{
PREVALENCIA Y EVOLUCIÓN CLÍNICA DE PACIENTES CON HERIDAS POR ARMA DE FUEGO EN LA COLUMNA VERTEBRAL
}

\author{
PREVALÊNCIA E EVOLUÇÃO CLÍNICA DE PACIENTES COM FERIMENTOS POR ARMA \\ DE FOGO NA COLUNA VERTEBRAL
}

\author{
PREVALENCE AND CLINICAL COURSE OF PATIENTS WITH GUNSHOT \\ WOUNDS TO THE SPINE
}

José María Jiménez Avila¹, Irving Hiram Ramos Díaz

\section{RESUMEN}

Introducción: Objetivos: Determinar la prevalencia, mortalidad y evolución clínica de los pacientes con heridas por arma de fuego en columna vertebral y determinar si la extracción del proyectil mejora el estado neurológico de los pacientes. Método: Se realizó un estudio de serie de casos, donde se analizaron los registros de todos los pacientes con heridas por arma de fuego en la columna vertebral ingresados en la UMAE, Hospital de Especialidades del CMNO del IMSS, de septiembre del 2010 a septiembre del 2011, que eran compatibles con los criterios de inclusión, exclusión y de no inclusión. Se midió el grado de discapacidad de Ios pacientes mediante la escala de Oswestry. Resultados: El grupo se formo por 13 (86,7\%), pacientes del sexo masculino y 2 $(13,3 \%)$ del sexo femenino, con edad media de 42 años y desviación estándar de 14 años. Las regiones más afectadas fueron torácica 7 (46,7\%), cervical 5 (33,3\%) y por último, lumbar con $3(20 \%)$ pacientes; la estancia intrahospitalaria observada con mayor frecuencia fue $7(13,3 \%)$ y $10(13.3 \%)$ días, con 2 casos cada una; la estancia hospitalaria mínima fue de 3 días, con tan solo un $(6,7 \%)$ caso y la mayor fue de 94 días, en 1 (6,7\%) paciente. La estancia superior a 10 días ocurrió en 11 (73,3\%) pacientes y la inferior a 10 días, en 4 (26,7\%) pacientes. El tratamiento quirúrgico se realizó en 4 (26,7\%) pacientes y 11 (73,3\%) pacientes solo recibieron tratamiento clínico; la extracción del proyectil se efectúo en $3(20 \%)$ pacientes, de los cuales 2 tuvieron infección. Se observaron complicaciones en 14 (93,3\%) pacientes y tan solo en 1 (6,7\%) no hubo complicaciones. En 6 (40\%) pacientes ocurrió infección en comparación con $9(60 \%)$ en quienes no la presentaron. El estatus neurológico mediante la escala de ASIA inicial predominante fue "A" en 7 (46,7\%) y el ASIA final predominante fue "B" en 6 (40\%) casos. Fallecieron 3 (20\%) pacientes y $12(80 \%)$ pacientes sobrevivían durante el periodo del estudio. El grado de discapacidad medido mediante la escala de Oswestry inicial mínimo fue de 18 puntos, el máximo de 94, con media de 62 y desviación estándar de 22.2. El Oswestry final tuvo una media de 58 puntos con desviación estándar de 23.5. Conclusiones: En nuestro estudio podemos concluir que las heridas por arma de fuego en la columna, ocasionan un alto grado de discapacidad y evolucionan con alta morbilidad. A esto hay que añadir que tanto el tratamiento clínico cuanto el quirúrgico, no proporcionan mejoría sustancial en el estado neurológico y funcional del paciente. Tan solo se limitan a medidas de soporte para disminuir en lo posible las complicaciones, que a final de cuenta, son las que influenciarán en la calidad y sobrevida de los afectados. La extracción del proyectil y el tiempo transcurrido desde la cirugía no alteran el estado funcional final del paciente.

Descriptores: Arma de fuego; Columna vertebral; Pronóstico; Prevalencia; Evolución clínica.

\section{RESUMO}

Objetivos: Determinar prevalência, mortalidade e evolução clínica dos pacientes com ferimentos por arma de fogo na coluna vertebral e determinar se a extração do projétil melhora o estado neurológico dos pacientes. Método: Realizou-se um estudo de série de casos por meio da análise dos prontuários médicos de todos os pacientes com ferimentos por arma de fogo na coluna vertebral, internados na UMAE, Hospital de Especialidades do CMNO do IMSS, de setembro de 2010 a setembro de 2011, que eram compatíveis com os critérios de inclusão, exclusão e não-inclusão. O grau de incapacidade dos pacientes foi medido pelo índice de Oswestry. Resultados: O grupo foi formado por 13 (86,7\%) pacientes do sexo masculino e 2 (13,3\%) do sexo feminino, com média de idade de 42 anos e desvio padrão de 14 anos. As regiões mais afetadas foram torácica 7 (46,7\%), cervical 5 (33,3\%) e, por último, lombar, co 3 (20\%) pacientes; a permanência no hospital observada com mais frequência foi 7 (13,3\%) e 10 (13,3\%) dias com 2 casos cada uma; a permanência hospitalar mínima foi de 3 dias, com apenas um (6,7\%) caso e a maior foi de 94 dias, em um (6,7\%) paciente. A estadia superior a 10 dias ocorreu em 11 (73,3\%) pacientes e a inferior a 10 dias, em 4 (26,7\%). O tratamento cirúrgico foi realizado em 4 (26.7\%) pacientes e 11 (73,3\%) pacientes receberam apenas tratamento clínico; a extração do projétil foi executada em 3 (20\%) pacientes, dos quais, dois tiveram infecção. Foram constatadas complicações em 14 (93.3\%) pacientes e apenas um (6,7\%) não as apresentou. Em 6 (40\%) pacientes verificou-se infecção, em comparação com 9 (60\%) que não tiveram. O estado neurológico pela escala ASIA inicial foi predominante "A" em 7 (46,7\%) e a escala ASIA final foi predominante "B" em 6 (40\%) casos. Três (20\%) pacientes foram a óbito e 12 (80\%) estavam vivos durante o período do estudo. O grau de incapacidade medido pelo índice de Oswestry inicial mínimo foi 18 pontos e o máximo foi 94, com média de 62 e desvio padrão de 22,2. O índice de Oswestry final teve média de 58 pontos, com desvio padrão de 23,5. Conclusões: Em nosso estudo, podemos concluir que os ferimentos por arma de fogo na coluna vertebral, ocasionam alto grau de incapacidade e evoluem com alta morbidade. É preciso acrescentar que tanto o tratamento clínico quanto o cirúrgico,

1. Cirujano Ortopédico, Servicio de Ortopedia del Centro Médico Nacional de Occidente, IMSS en Guadalajara, Jalisco, México.

Trabalho realizado no Servicio de Ortopedia del Centro Médico Nacional de Occidente, IMSS en Guadalajara, Jalisco, México.

Correspondência: Puerta de Hierro Medical Center, Av. Empresarios núm. 150, Piso 23, Colonia Puerta de Hierro, Zapopan, Jalisco, México. josemajimeneza@ hotmail.com

Recibido 22/03/2012, aceptado 09/11/2012 
não proporcionam melhora substancial do estado neurológico e funcional do paciente. Ambos limitam-se a medidas de apoio para reduzir o máximo possível de complicações, que por fim, são o que influenciarão a qualidade e a sobrevida dos afetados. A extração do projétil e o tempo transcorrido desde a cirurgia não alteram o estado funcional final do paciente.

Descritores: Arma de fogo; Coluna vertebral; Prognóstico; Prevalência; Evolução clínica.

\begin{abstract}
Objectives: To determine prevalence, mortality and clinical course of patients with gunshot wounds of the spine and determine if the extraction of the bullet improves their neurological status. Methods: We conducted a case series study by analyzing the medical records of all patients with gunshot wounds in the spine, hospitalized in UMAE, Specialties Hospital of the CMNO, IMSS, from September 2010 to September 2011, which were compatible with the inclusion, exclusion, and not-inclusion criteria. The degree of disability of patients was measured by the Oswestry index. Results: The group consisted of 13 (86.7\%) males and 2 (13.3\%) females, with a mean age of 42 years and standard deviation of 14 years. The regions most affected were thoracic with 7 (46.7\%), cervical with 5 (33.3\%) and, finally, lumbar, with 3 (20\%) patients; the most frequent hospital stays were 7 (13, 3\%) and 10 (13.3\%) days with 2 cases each; minimum hospital stay was 3 days, with only one (6.7\%) case and the largest was 94 days in one (6.7\%) patient. A stay exceeding 10 days occurred in 11 (73.3\%) patients and less than 10 days in 4 (26.7\%). Surgical treatment was performed in 4 (26.7\%) patients and 11 (73.3\%) patients received only medical therapy; the extraction of the bullet was performed in 3 (20\%) patients, of whom two had infection. Complications were observed in 14 (93.3\%) patients and one (6.7\%) did not. Six (40\%) patients had infection in comparison with nine (60\%) who had not. The neurological status by the initial ASIA scale was predominantly "A" in 7 (46.7\%) and the final ASIA scale was predominantly "B" in $6(40 \%)$ cases. Three (20\%) patients died and 12 (80\%) were alive during the study period. The degree of disability measured by the initial Oswestry index was 18 points minimum and maximum was 94, with a mean of 62 and a standard deviation of 22.2. The final Oswestry index averaged 58 points, with a standard deviation of 23.5. Conclusions: In our study, we conclude that the injury by firearm in the spine causes a high degree of disability and evolve with high morbidity. It should be added that both the medical and the surgical treatment do not provide substantial improvement in neurological and functional status of the patient. Both are limited to support actions to reduce the possible complications, which ultimately is what will influence the quality and survival of those affected. The extraction of the bullet and the time elapsed since surgery does not change the final functional status of the patient.
\end{abstract}

Keywords: Wounds, Gunshot; Spine; Prognosis; Prevalence; Clinical evolution.

\section{INTRODUCCIÓN}

Los efectos de las lesiones en la columna vertebral producidas por armas de fuego en la población civil merecen atención epidemiológica con el fin de conocer las circunstancias en las que se producen y actuar en consecuencia ${ }^{1}$.

En la actualidad las heridas por proyectil de arma de fuego ocupan aproximadamente el 13\% de todos los casos de lesión al cordón medular y/o raquídeo aumentando a $14 \%$ si se toman en cuenta todas las heridas penetrantes (arma blanca, punzo- cortantes, etc.) En la literatura se habla de que más del $60 \%$ de los casos ocurren en edades entre 15 y 29 años y otro $23 \%$ en pacientes de 30 a 34 años $^{2-4}$.

La región más frecuentemente afectada es la torácica con aproximadamente $45 \%$, la sigue la región lumbar con un 30\% y en último lugar está la cervical con 25\%5,6.

Las heridas por proyectil de arma de fuego ocupan el tercer lugar como causa de lesiones traumáticas de columna un poco por debajo de los accidentes de tráfico y las caídas, afectando principalmente a población joven y económicamente productiva.

Los efectos de las lesiones en la columna vertebral producidas por armas de fuego en la población civil merecen atención epidemiológica con el fin de conocer las circunstancias en las que se producen y actuar en consecuencia, por lo que resulta imperativo conocer el estado real de la situación en nuestra población de pacientes debido al alto grado de discapacidad y mortalidad de estos pacientes.

A pesar de que han mejorado las técnicas quirúrgicas y el acceso a los servicios médicos, no se han observado mayores cambios en el estado neurológico ni el grado de discapacidad en los pacientes con lesiones espinales por arma de fuego esto debido a la falta de un consenso o un manejo protocolizado para el manejo de dichas lesiones, por lo que el presente trabajo pretende dar a conocer un panorama para mejorar el tratamiento otorgado y disminuir el grado de discapacidad, así como la morbimortalidad asociada a estas lesiones por arma de fuego 1,4,7-9.

El objetivo primario de la evaluación inicial se debe dirigir a identificar las lesiones que comprometan la vida, el mantenimiento de la vía aérea, la respiración y circulación son los elementos más importantes durante el periodo inicial $\left.\right|^{6,8,10}$.

La evaluación del paciente inicia con una exploración física detallada, el proyectil, al igual que un examen neurológico minucioso es obligatorio, el saber la trayectoria del proyectil aportara valiosa información ya que orientara indicios que estructuras u órganos pueden estar lesionados y esto es un factor pronostico de posibles complicaciones y grado de recuperación que tendrá el afectado6-8,10.

Se realiza inmovilización de la columna cervical a los heridos por arma de fuego en columna, en especial aquellos que presentan HPPAF en cabeza y cuello, con escala de Glasgow menor de 8, déficit neurológico, alteración del estado mental y con afección medular ${ }^{10,14}$.

Mención especial merece a las heridas por arma de fuego en cuello, ya que el uso rutinario de collarín rígido no está justificado, debido al a baja frecuencia de fracturas de vertebras cervicales y / o de inestabilidad de estas lesiones ${ }^{11,13,14}$.

La columna torácica es el área más comúnmente afectada, se debe evaluar daño al corazón, pulmones y grandes vasos, el abdomen también deberá ser evaluado en búsqueda de cualquier daño a órganos sólidos, lesiones del intestino o de grandes vasos, ya que estas lesiones tienen un alto riesgo de infección a la columna, si no son tratados con antibióticos de manera adecuada ${ }^{9}$.

Las heridas por arma de fuego en columna resultan comúnmente en lesiones completas, que es la pérdida completa de la función motora y la sensibilidad distal a la lesión de la médula espinal, algunas series de estudios reportan $62 \%$ de los casos con paraplejia completa, mientras que otros sugieren $29 \%$ con paraplejia completa, 23\% paraparesia incompleta y $19 \%$ cuadriplejia completa, en algunos casos pueden producir una lesión incompleta que representa un déficit distal a la lesión de la médula espinal, pero con preservación de algunas funciones ${ }^{15-17}$.

Como con todos los pacientes en los que se sospecha o se ha producido una lesión de columna vertebral, se deben obtener radiografías simples anteroposterior y lateral, el nivel de cualquier 
fractura de la columna asociados debe ser identificado, así como la ubicación aproximada de las balas o fragmentos retenidos ${ }^{6,8,10}$.

La inestabilidad estructural de la columna es poco frecuente después de una lesión penetrante, se pueden realizar radiografías dinámicas a juicio del médico, las cuales pueden ser útiles cuando la estabilidad siga siendo cuestionable $e^{10,13,14}$

La TAC es el estudio de elección para estas lesiones, ya que permite determinar las características de la fractura y localización del proyectil y demuestra claramente la trayectoria, la dispersión de la imagen y artefactos de retención de fragmentos metálicos, puede ser problemático pero en general no oculta líneas de fractura de manera significativa utilizando técnicas modernas y de pequeños cortes en las áreas de interés, las imágenes transversas usualmente son apropiadas para realizar un diagnostico adecuado ${ }^{10}$ (Figura 1 ).

El uso de la resonancia magnética es controversial, es muy útil en la evaluación de la médula, la pronta realización de esta, en los casos de lesión completa de la médula, tiene una enorme implicación pronostica, puede ocurrir migración de la bala por la fuerza del magneto y posiblemente, puede llevar a mayor daño neurológico o de los tejidos blandos, el uso de la resonancia magnética se debe individualizar de acuerdo a las necesidades específicas de cada paciente ${ }^{6-8,10}$ (Figuras 2 y 3 ).

El tratamiento de las heridas por arma de fuego en columna, deberá ser individualizado basado en la edad, el nivel y tipo de lesión, la progresión del deterioro neurológico y la presencia de otras lesiones asociadas ${ }^{6}$

Se debe realizar profilaxis contra el tétanos en todos los casos con heridas por arma de fuego en columna de manera inmediata desde el momento que el paciente ingresa al área de urgencias. (toxoide tetánico $0.5 \mathrm{ml} \mathrm{IM}$ ) e inmunoglobulina antitetánica ${ }^{6,10}$.

Se iniciará tratamiento antimicrobiano de amplio espectro de manera inmediata, de acuerdo al área anatómica involucrada y las lesiones concomitantes asociadas; el cuál se deberá de continuar por 48 a 72 hrs, en los casos que exista perforación de vísceras intraabdominales y de manera particular, afección del colon, se deberá dar tratamiento antimicrobiano de amplio espectro con cobertura para Gram positivos, Gram negativos y anaerobios durante un periodo no menor de 7-14 días.

Utilizando este protocolo, la incidencia de infecciones en columna o de meningitis, se mantiene en el rango de 0 a $2 \%$, incluso en ausencia de desbridamiento quirúrgico, de manera alterna en otros estudios realizados se reporta que el retiro de la bala y el desbridamiento quirúrgico estuvo asociado a una incidencia 6 veces mayor de riesgo de infección comparado con el simple uso de antibióticos de manera aislada ${ }^{6,10}$

A excepción de indicaciones tales como el deterioro neurológico o la toxicidad del plomo, la extracción de la bala no se recomienda

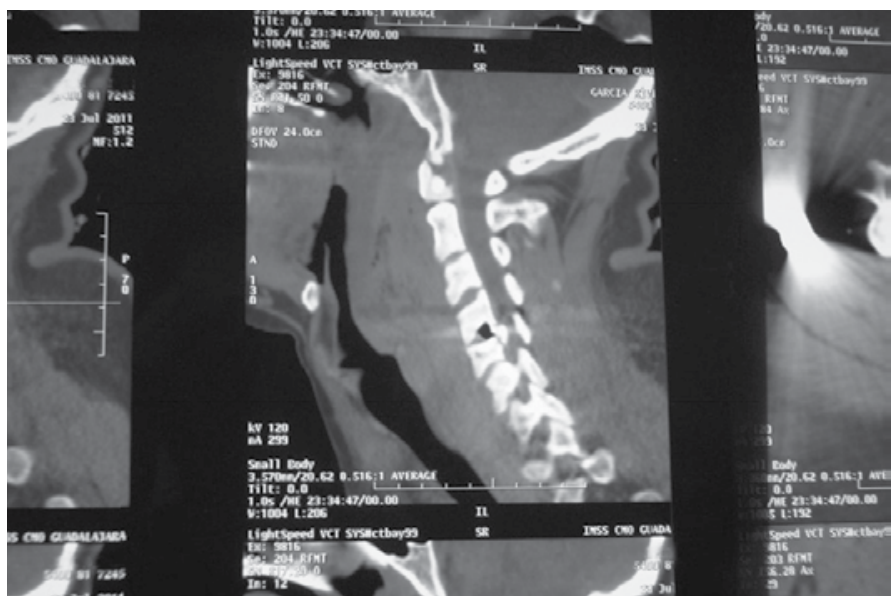

Figura 1. Proyectil dentro del canal medular en el segmento C4-C5.
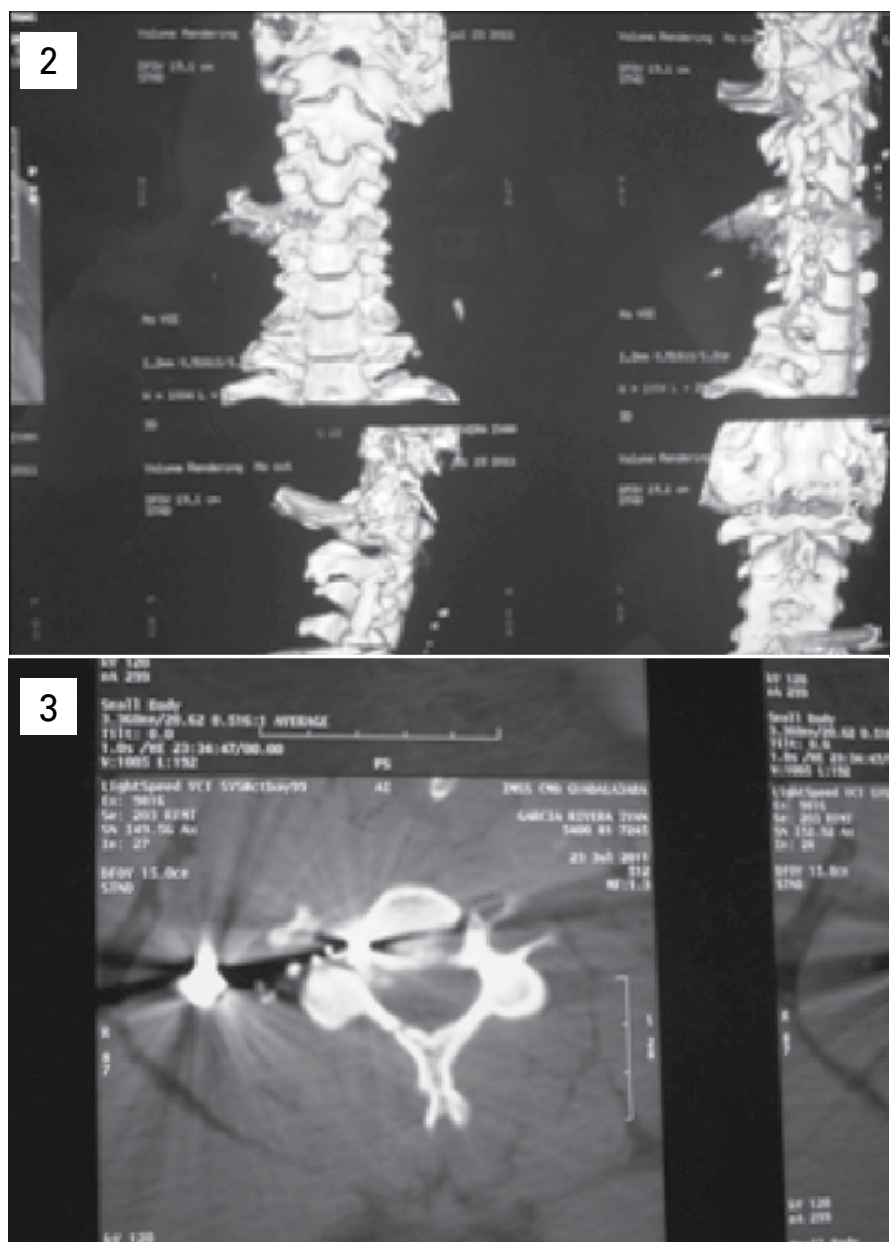

Figuras 2 y 3. Trayectoria del proyectil alojado en el cuerpo vertebral.

para disminuir el riesgo de infección.

Algunos estudios han demostrado que no hay evidencia de mejoría en el estado neurológico con el tratamiento con esteroides, pero si incrementa el riesgo de infecciones y complicaciones gastrointestinales ${ }^{8,10}$

Otros autores mencionan que las balas y los fragmentos de ellas que estén dentro del canal medular deberán ser extraídos solo si estos son fácilmente accesibles, otro hecho que apoya este manejo son los escasos reportes de complicaciones tardías por la retención de proyectiles tales como migración de estos o datos de toxicidad, se deberá dar seguimiento a los fragmentos retenidos con estudios de imagen de manera periódica y medición de los niveles séricos de plomo y en caso necesario iniciar una terapia de que lesión de este elemento6,8-10.

El dolor debe ser manejado agresivamente, el uso ocasional de analgésicos neuroaxiales, se realizará después de evaluar el tipo de lesión y la estabilidad hemodinámica del paciente, aunque la proporción de pacientes tratados quirúrgicamente con lesiones penetrantes de la columna vertebral varía ampliamente en la literatura, la mayoría de los pacientes no requieren intervención quirúrgica ${ }^{6,10}$.

Hay poca evidencia para apoyar la descompresión quirúrgica para la mayoría de los pacientes con déficit neurológico establecido, varios estudios han demostrado que no hay diferencia significativa en los resultados neurológicos entre los pacientes que se sometieron a laminectomía descompresiva y aquellos que se dio tratamiento no quirúrgico, además en el grupo quirúrgico se observa una mayor tasa de complicaciones tales como fistulas de liquido cefalorraquídeo y meningitis ${ }^{6-8,10,19}$ 


\section{MATERIAL Y MÉTODOS}

Se realizo un estudio de serie de casos donde se donde se analizaron los expedientes de todos los pacientes con heridas por arma de fuego en columna vertebral ingresados en la Unidad Médica de Alta Especialidad, Hospital de Especialidades del CMNO del IMSS que reunían con los criterios de inclusión, tales como ser pacientes hospitalizados, mayores de 18 años, de ambos sexos con herida por arma de fuego con afección a columna vertebral, que contaran con expediente completo en el archivo clínico, se excluyeron aquellos que no contaran con expediente completo y que la lesión estuviera acompañada de una lesión en cráneo que le provocara déficit neurológico.

Del expediente se tomaron en cuenta variables tales como, la edad, sexo, el tipo de tratamiento, los días de estancia intrahospitalaria, la extracción o no del proyectil, la presencia de complicaciones, y el estado neurológico.

\section{RESULTADOS}

Del periodo comprendido de septiembre del 2010 a septiembre del 2011, se estudiaron un total de 15 pacientes del Hospital de Especialidades del Centro Médico Nacional de Occidente, en Guadalajara Jalisco, los cuales presentaron heridas por arma de fuego en columna vertebral.

El grupo general se formo por $13(86.7 \%)$, pacientes del sexo masculino y 2 (13.3\%) del sexo femenino, con una edad promedio de 42 y una d.s. de 14 años.

El sector anatómico de la columna vertebral con mayor afectación fue el torácico 7 (46.7\%), seguido del cervical 5 (33.3\%) y por último el lumbar con 3 (20\%) pacientes, la estancia intrahospitalaria con mayor frecuencia observada fue de 7 (13.3\%) y 10 (13.3\%) días con 2 casos cada uno. En 1 (6.7\%) paciente se presentó una estancia hospitalaria mínima de 3 días, siendo la mayor de 94 días.

El tratamiento quirúrgico se realizó en 4 (26.7\%) pacientes y en 11 (73.3\%) pacientes solo recibieron tratamiento médico, en quienes se otorgó tratamiento quirúrgico 2 (66.7\%) pacientes, este se realizo a los 11 días posteriores a la lesión y en 1 (33.3\%) paciente se realizó transcurrido 17 días de la lesión, la extracción del proyectil se efectúo en 3 (20\%) pacientes contrario a 12 (80\%) en quienes no se llevo a cabo. (Figuras 4 y 5).

Se observaron complicaciones en 14 (93.3\%) pacientes y tan solo en 1 (6.7\%) no ocurrieron, en 6 (40\%) pacientes ocurrió infección comparado contra 9 (60\%) en quienes no la presentaron.

El estatus neurológico mediante la escala de ASIA inicial predominante fue "A" en 7 (46.7\%), seguido del "B" en 5 (33.3\%) pacientes y el ASIA final predominante fue el "B" en 6 (40\%) 'pacientes, seguido del "A" en $5(33.3 \%)$ casos.

El fallecimiento ocurrió en 3 (20\%) pacientes y 12 (80\%) pacientes sobrevivían durante el tiempo que se realizo el estudio.

El grado de discapacidad medido mediante la escala de Oswestry inicial mínimo fue de 18 puntos, el máximo de 94, con una media de 62.1, y una d.s. de 22.2, siendo el Oswestry final de 58 puntos con una d.s. de 23.5 (Tabla 1)

\section{DISCUSIÓN}

La prevalencia de las lesiones traumáticas de columna se ha estimado entre 30 a 40 casos por millón de habitantes al año, de estas, las heridas por arma de fuego en columna ocupan el 3er lugar, con aproximadamente 13 a 17\% de los casos, solo detrás de los accidentes de tráfico y las caídas.

Durante el periodo de septiembre del 2010 a septiembre 2011 en el Hospital de Especialidades del Centro Médico Nacional de Occidente, se contabilizaron 235 casos de lesiones traumáticas de columna, de las cuales, se identificaron 15 casos, correspondiendo al $6.38 \%$ de los casos, esto muestra una prevalencia menor a lo
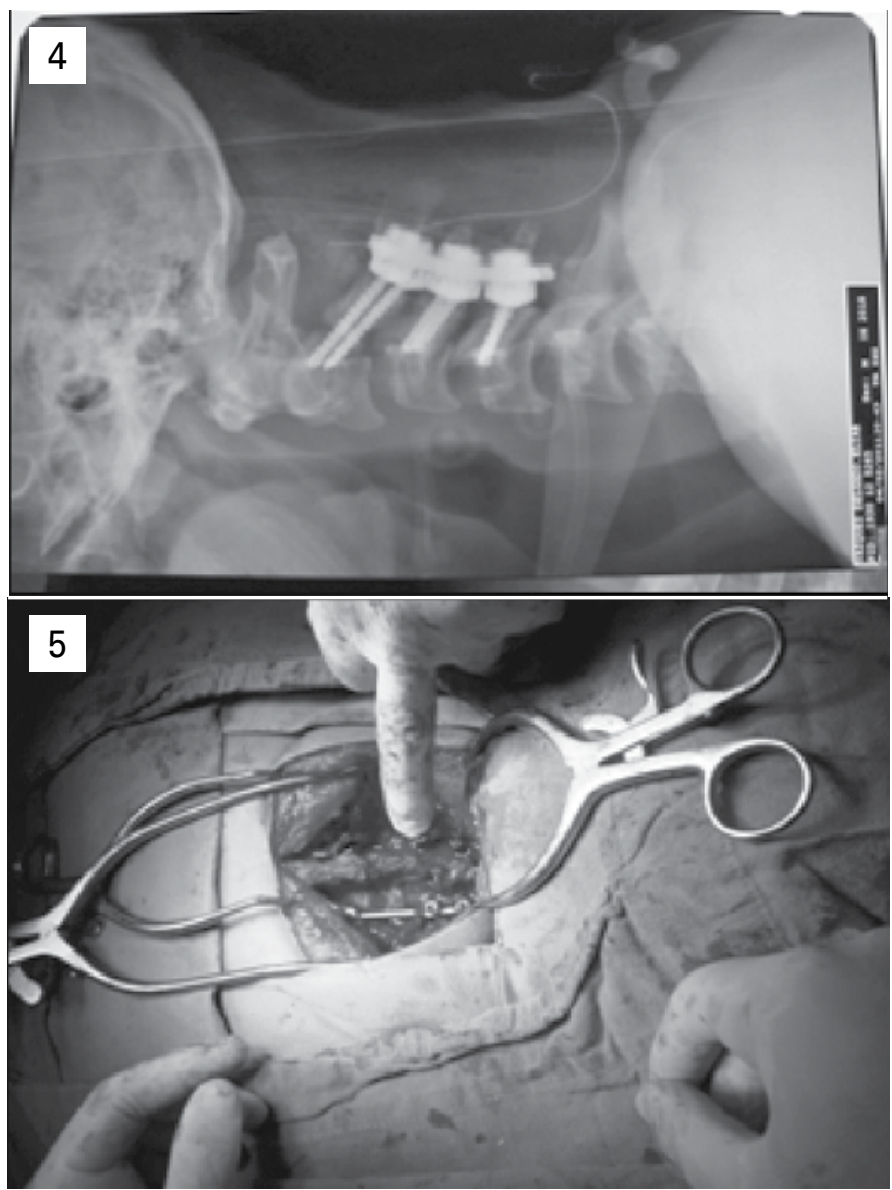

Figuras 4 y 5. Instrumentación posterior por inestabilidad provocada por la herida por proyectil arma de fuego.

esperado de acuerdo a la literatura existente.

De los 11 (73\%) pacientes que presentaron estancia intrahospitalaria > 10 días, el 100\% presento infección, mientras que el grupo con estancia intrahospitalaria < 10 dias , 4 (26.7\%) ninguno de los pacientes sufrió infección, lo cual demuestra la relación que existe entre la mayor estancia intrahospitalaria, y el mayor riesgo de infección tal como lo describe Buxton.

Respecto al tratamiento quirúrgico, este se encontraba relacionado con el incremento en la mortalidad y las complicaciones, aunque no existe un consenso del tiempo ideal para realizar el tratamiento quirúrgico, se recomienda realizarlo antes de las 2 semanas, ya que después de este tiempo se ha encontrado mayor incidencia de infecciones que dificultan y ensombrecen el resultado del tratamiento quirúrgico, sin que exista una mejoría del estado neurológico o funcional, pero si un incremento en el riesgo de mortalidad $^{1,6,10,12}$.

En nuestra población de estudio a 3 (20\%) pacientes se les realizó la extracción del proyectil, 2 presentaron infección y uno falleció, sin que esto produjera una mejoría en el estado neurológico, pero si incrementó las complicaciones, por lo tanto no se recomienda la extracción del proyectil de manera rutinaria, solo si se piensa realizar tratamiento quirúrgico y el acceso mediante el abordaje ofrece la extracción del mismo sin complicaciones.

\section{CONCLUSIONES}

Las heridas por arma de fuego en columna, ocasionan un alto grado de discapacidad y cursan con una alta morbilidad, a esto hay que añadir que tanto el tratamiento médico y quirúrgico, no proporcionan una mejoría sustancial en el estado neurológico y 
Tabla 1. Datos demográficos de los pacientes con herida por proyectil arma de fuego.

\begin{tabular}{|c|c|c|c|c|c|c|}
\hline Variables & & Frec Abs. & Frec Relat. & Variable & Media & D.S. \\
\hline \multirow[t]{2}{*}{ Sexo } & Masculino & 13 & $86.70 \%$ & Edad & 42.0667 & 13.99 \\
\hline & Femenino & 2 & $13.30 \%$ & Días Estancia & 26.5333 & 25.9611 \\
\hline \multirow[t]{2}{*}{ Sector } & cervical & 5 & $33.33 \%$ & Oswestry Inicial & 62.1333 & 22.2514 \\
\hline & Lumbar & 3 & $20 \%$ & & & \\
\hline \multirow[t]{2}{*}{ Tratamiento } & Quirúrgico & 4 & $26.70 \%$ & & & \\
\hline & No quirúrgico & 11 & $73.30 \%$ & & & \\
\hline Extracción & No quirúrgico & 12 & $80 \%$ & & & \\
\hline \multirow[t]{2}{*}{ Complicaciones } & $\mathrm{Si}$ & 14 & $93.30 \%$ & & & \\
\hline & No quirúrgico & 1 & $6.70 \%$ & & & \\
\hline \multirow[t]{2}{*}{ Infección } & $\mathrm{Si}$ & 6 & $40 \%$ & & & \\
\hline & No & 9 & $60 \%$ & & & \\
\hline \multirow[t]{2}{*}{ ASIA inicial } & A & 7 & $46.70 \%$ & & & \\
\hline & $E$ & 1 & $6.70 \%$ & & & \\
\hline \multirow[t]{5}{*}{ ASIA final } & A & 5 & $33.30 \%$ & & & \\
\hline & $B$ & 6 & $40.00 \%$ & & & \\
\hline & $\mathrm{C}$ & 2 & $13.30 \%$ & & & \\
\hline & $\mathrm{D}$ & 1 & $6.70 \%$ & & & \\
\hline & $E$ & 1 & $6.70 \%$ & & & \\
\hline \multirow[t]{2}{*}{ Vive } & $\mathrm{Si}$ & 12 & $80 \%$ & & & \\
\hline & No & 3 & $20 \%$ & & & \\
\hline \multirow[t]{2}{*}{ Días estancia } & $<10$ días & 4 & $26.70 \%$ & & & \\
\hline & $>10$ días & 11 & $73.30 \%$ & & & \\
\hline Días previos & D 11 & $200.00 \%$ & $66.70 \%$ & & & \\
\hline
\end{tabular}

funcional del paciente, tan solo se limita a dar medidas de soporte para disminuir en lo posible las complicaciones, que a final de cuenta, dichas acciones son las que incidirán en la calidad y sobrevida de los afectados, por lo tanto en base a los resultados obtenidos podemos concluir que existen pocos reportes sobre el tiempo oportuno para realizar el tratamiento quirúrgico, algunos autores mencionan entre el quinto y decimo día después de la lesión como el momento ideal para la cirugía, se ha encontrado una mayor incidencia de infección y aracnoiditis en aquellos casos que fueron sometidos a cirugía 2 semanas después de la lesión 6,7,10,19.

\section{REFERÊNCIAS}

1. Turgut M, Ozcan O. E, Gürçay O, Saglam S. Civilian penetrating spinal firearm injuries of the spine. Results of surgical treatment.Arch Orthop Trauma Surg. 1994;113(5):290-3.

2. Rodriguez R, Anaya S, Fajardo R, Vazquez-Vela G. Heridas por proyectil de arma de fuego en columna sin lesión medular. Presentación de dos casos. Rev Mex Ortop Traum. 2000;14(1):96-8.

3. Smith W, Simmonds J, Alam Z, Grant R. Spinal cord Injury caused by gunshot wounds: the cost of rehabilitation. Clin Orthop Relat Res. 2003;(408):145-51.

4. López B, Anaya S, Rodriguez R, Palapa R. Estudio clínico epidemiológico de pacientes con lesiones de la columna vertebral por herida de bala atendidos en un hospital de referencia de seguridad social. Rev Mex Ortop Traumatol. 2000;14(1):44-7.

5. Azevedo-Filho H, Martins C, Carneiro-Filho G, Azevedo R, Azevedo F. Gunshot wounds to the spine: study of 246 patients. Arq Neuro-Psiquiatr. 2001;59(3a):645-6.

6. Heary R, Kumar S. Treatment of civilian gunshot wounds for the spine.Techn Orthop. 2006;21(3):205-13.

7. Duz B, Cansever T, Secer HI, Kahraman S, Daneyemez MK, Gonul E.Evaluation of spinel missile injuries with respect to bullet trajectory surgical indications and timing of surgical intervention. Spine (Phila Pa 1976). 2008:33(20):E746-53.

8. Buxton N. The militar medical management of missile injury to the spine: a review of the literature and proposal of guidelines. J R Army Med Corps. 2001;147(2):168-72

9. Orndorff DG, Shen FH. Small -caliber gunshot wound with fragment lodged in thoracic foramen in a patient with partial Brown- sequard syndrome. Am J Orthop (Belle Mead NJ). 2008;37(3):E55-8
10. Potter K, Groth A, Kuklo T. Penetrating thoracolumbar spine injuries. Cur Opin Orthop. 2005;16(3):163-8

11. Dalgic A, Okay O, Nacar O, Daglioglu E, Pasaoglu L, Belen D. Vertebral artery insult at the transverse foramina by gun shot wounds: report of two cases. Turk Neurosurg. 2009;19(4):413-6.

12. Lanoix R, Gupta R, Leak L, Pierre J. Spine injury associated with gushot wounds to the head: retrospective study and literatura review. J Trauma. 2000;49:860-3.

13. Medzon R, Rothenhaus T, Bono C, Grindinger G, Rathlev N. Stability of cervical spine fractures after gunshot wounds to the head and neck. Spine (Phila Pa 1976). 2005;30(20):2274-9.

14. Lustenberger T, Talving P, Lam L, Kobayashi L, Inaba K, Plurad D. Unstable cervical spine fracture after penetrating neck injury: A rare entity in an analysis of 1069 patients. J Trauma. 2011;70(4):870-2

15. Quigley KJ, Place HM. The role of debridement and antibiotics in gunchost wounds to the spine. J Trauma. 2006;60(4):814-9

16. Lim MR, Lee JY, Vaccaro AR. Surgical infections in the traumatized spine. Clin Orthop Relat Res. 2006;444:114-9.

17. Bagley LJ. Imaging of spinal trauma. Clinics of North Am 2006:44(1):1-12.

18. Kalkan E, Keskin F, Cengiz SL, Baysefer A. A case report of firearm bullet settling into the thoracic spinal canal without causing neurological deficit or vertebral bone destruction. Arch Orthop Trauma Surg. 2007;127(8):637-41

19. Mirovsky Y, Shalmon E, Blankstein A, Halperin N. Complete paraplegia following gunshot injury without direct trauma to the cord. Spine (Phila Pa 1976). 2005;30(21):2436-8. 